\title{
Suppression of ballistic helical transport by isotropic dynamical magnetic impurities
}

\author{
Oleg M. Yevtushenko*1 and Vladimir I. Yudson ${ }^{2,3}$ \\ ${ }^{1}$ Institut für Theorie der Kondensierten Materie, \\ Karlsruhe Institute of Technology, 76128 Karlsruhe, Germany \\ ${ }^{2}$ Laboratory for Condensed Matter Physics, National Research University Higher School of Economics, Moscow, 101000, Russia \\ ${ }^{3}$ Russian Quantum Center, Skolkovo, Moscow Region 143025, Russia
}

(Dated: July 2, 2021)

\begin{abstract}
Dynamical magnetic impurities (MI) are considered as a possible origin for suppression of the ballistic helical transport on edges of 2D topological insulators. The MIs provide a spin-flip backscattering of itinerant helical electrons. Such a backscattering reduces the ballistic conductance if the exchange interaction between the MI and the electrons is anisotropic and the Kondo screening is unimportant. It is well-known that the isotropic MIs do not suppress the helical transport in systems with axial spin symmetry of the electrons. We show that, if this symmetry is broken, the isotropic MI acquires an effective anisotropy and suppresses the helical conductance. The peculiar underlying mechanism is a successive backscattering of the electrons which propagate in the same direction and have different energies. The respective correction to the linear conductance is determined by the allowed phase space of the electrons and scales with temperature as $T^{4}$. Hence, it disappears at small temperatures. This qualitatively distinguishes effects governed by the MIs with the induced and bare anisotropy; the latter is the temperature independent. If $T$ is smaller than the applied bias, finite $\mathrm{eV}$, the allowed phase space is provided by the bias and the differential conductance scales as $(e V)^{4}$.
\end{abstract}

\section{INTRODUCTION}

Suppression of the edge transport in two-dimensional (2D) topological insulators [1-4] has attracted considerable attention of the community and remains the hot topic even after a decade of the intensive research because of the absence of the fully self-consistent theoretical explanation. The time-reversal symmetry and the non-trivial topology of the bulk guarantee helicity (lockin relation between spin and direction of propagation) of the gapless one dimensional (1D) edge modes [5-7]. Helicity prohibits an elastic single-particle backscattering by a spinless potential. Thus, at least in the absence of interactions, the helical modes are not liable to effects of material imperfections, e.g. localization. Many physical mechanisms, which are beyond the simplest single-particle picture and can suppress the helical conductance, have been suggested aiming to explain experimental data: multi-particle backscattering [8, 9], electron-electron/phonon interactions and inelastic scattering of the helical electrons [10-16], and their exchange interaction with nanomagnets $[17,18]$ or localized dynamical magnetic moments - magnetic impurities (MI), [19-30] to name just a few. We focus on the latter mechanism below.

An exchange interaction of the helical electrons with the MIs can result in energy-preserving backscattering accompanied by the spin-flip. That is why the MIs were considered as a serious obstacle for the ballistic helical transport in the topological insulators even with the spin axial symmetry of the electrons [31]. As a matter of fact, the MIs, which are isotropically coupled to the itinerant electrons (the isotropic MIs for brevity), cannot themselves break the spin U(1) symmetry and, therefore, cannot influence the dc conductance [32], see also Refs.
[21, 22, 33, 34]. The anisotropically coupled MIs (the anisotropic MIs for brevity) violate the spin conservation and are able to suppress the ballistic conductance but only if they are not Kondo screened. This requires either a high density of the MI array [21, 22, 33, 34], where the Kondo effect is overwhelmed by the MI correlations [35], or the temperature / the applied bias being larger than the Kondo temperature, $\max \{T,(e V)\}>T_{K}$, or a large value of the MI spin, $S>1 / 2[36-38]$.

The situation is crucially different in the topological insulators without axial spin symmetry of the electrons $[10,12,14]$. The absence of the spin symmetry can be caused, e.g., by the Rashba spin-orbit interaction, and does not contradict to the concept of the helical modes on the edges of the topological insulators [39]. In these systems, the helical electrons acquire a dependence of the spin orientation on their energy, the so-called spin texturing (ST) [40]. The well-known effect of the ST is the suppression of the helical conductance caused by the inelastic backscattering of the interacting electrons $[10,12]$. Another effect of the ST, which has not been addressed in the literature, is an influence of the isotropic (unscreened) MI on the helical transport of the noninteracting electrons. Since the scattering of a given electron by the MI does not change the electron energy, one could surmise that the ST is unimportant and the isotropic MI could not have any effect on the linear helical conductance similar to the model with spin axial symmetry [32]. This guess holds true but only at zero temperature.

We show in the present paper that, even in the absence of the electron interactions, the isotropic (unscreened) MI yields negative corrections to the dc differential helical conductance at finite temperatures, $e V<T$, or at a finite bias, $T<e V$. The underlying mechanism is an energy-preserving successive backscattering of the helical 


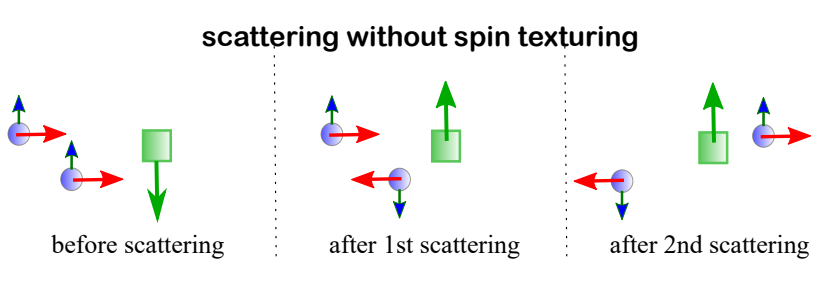

scattering with spin texturing
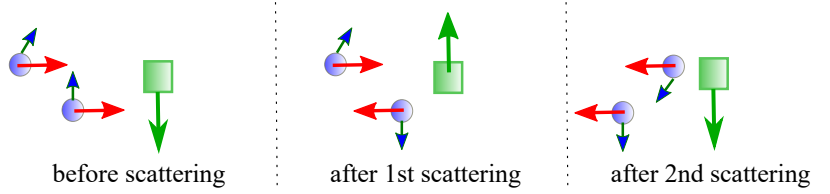

FIG. 1. Successive scattering of two right moving helical electrons (shaded circles), which have different energies, by the MI (shaded square), see the explanation in the text.

electrons which propagate in the same direction and have different energies. We elaborate this mechanism at qualitative and quantitative levels, derive the ST correction to the helical conductance, which scales in the proper limiting cases as $\delta G \propto \max \left\{T^{4},(e V)^{4}\right\}$, and compare our results with the suppression of the conductance caused by the anisotropic MI and by the combined effects of the ST and the electron interactions.

We would like to keep the discussion at the most transparent level and, therefore, use the representative example of the spin-1/2 MI weakly coupled to the noninteracting helical electrons at temperatures above $T_{K}$. The derivation of the differential conductance in this setup suffices to explain the combined effect of the MIs and the ST on helical transport. We touch upon more complicated situations, including Kosterlitz-Thoulesslike renormalizations of the MI-electrons coupling, very briefly and at a qualitative level.

\section{QUALITATIVE DISCUSSION}

The succesive backscattering of the helical electrons by the isotropic MI is illustrated by Fig. 1 where the cases with and without the ST are compared. Let us consider, for example, scattering of two right-moving helical electrons, see left panels of Fig.1.

If there is no ST, the MI spin becomes parallel to that of all incoming right-moving electrons already after the first backscattering. This blocks consecutive spin-flips and, therefore, the MI is unable to backscatter one after another two electrons, see the upper panel of Fig.1. Two electrons can be backscattered by the MI successively only if they have different chirality and, as a result, the dc helical conductance is not changed [32]. It is a wellknown example of systems where transport is ballistic despite backscattering of the individual electrons.

If, on the contrary, there is the ST, the first backscat- tering aligns the MI spin with that of the first incoming electrons before it is backscattered. Due to the ST, incoming electrons with another energy have different orientation of the spin. Therefore, the MI can backscatter two helical electrons of the same chirality one after another (see the lower panel of Fig.1) which results in the suppression of the ballistic helical conductance. This requires a finite energy shell of partially filled electron states around the Fermi energy. In the linear response regime, the allowed phase space of the electrons decreases with lowering temperature and the probability of the above described successive backscattering vanishes in the limit of small $T$.

Before presenting a more rigorous description, the scaling of the MI governed correction to the dc conductance can be deduced from a phenomenological approach. Expressions for the backscattering current and for the respective correction to the differential conductance read as follows:

$$
\mathcal{J}=e \partial_{t}\left(N_{R}-N_{L}\right)=2 e \partial_{t} N_{R}, \delta G=\partial_{V} \mathcal{J}
$$

where $e$ and $N_{R, L}$ are the electron charge and numbers of the right/left moving chiral electrons, respectively and we have taken into account the particle conservation law which relates chiral currents, $\mathcal{J}_{R}=-\mathcal{J}_{L}$. As we have already mentioned in Introduction, the backscattering caused by the isotropic MI cannot lead to the backscattering current if the spin axial symmetry is present. Breaking this symmetry leads to the ST and to the effective anisotropy of the MI. In the case of a weak ST, the anisotropic part of the coupling between the MI and the itinerant electrons is $\sim k^{2}$, with $k$ being the electron momentum [10]. The corresponding (leading in the electronMI coupling constant) contribution to $\mathcal{J} \propto \partial_{t} N_{R}$ is governed by the product of the external voltage multiplied by the square of the anisotropic part of the coupling constant, $\mathcal{J} \sim e V \times k^{4}$. The typical value of $k$ can be estimated as $k \sim \max \left\{T / v_{F}, e V / v_{F}\right\}$. Hence, one may expect $\delta G \sim T^{4} \mathcal{F}(e V / T)$, which is reduced to

$$
\delta G \propto \max \left\{T^{4},(e V)^{4}\right\},
$$

in corresponding limiting cases. Such an argumentation suggesting the scaling Eq.(2) is rather general and does not depend on the value of the MI spin at $\max \{T, e V\} \gg$ $T_{K}$. On the other hand, the above phenomenology is based on the intuition suggested by the Boltzmann kinetic equation, whose validity is a priory not clear. The kinetic equation must include distribution functions of the helical electrons and of the MI. The latter can be used only if the MI density matrix is diagonal. This is generically not correct in arbitrarily chosen basis [37] while the a proper basis is not know in advance, see calculations below. Besides, the power counting is unable to predict the scaling function $\mathcal{F}(e V / T)$ describing the crossover regime, $T \sim e V$, which might be of interest for comparing with experimental results. Hence, one needs a more rigorous theory of the backscattering current governed by the MI. We develop it in the next section by 
using the master equation approach for our main example of the spin-1/2 MI.

\section{MODEL AND METHOD}

Massless 1D fermions propagating on the edge of the 2D topological insulators are described by the standard Dirac Hamiltonian

$$
H_{0}=v_{F} \sum_{k} k\left(\Psi_{\mathrm{ch}}^{\dagger}(k) \hat{\sigma}^{z} \Psi_{\mathrm{ch}}(k)\right) .
$$

Here $\Psi_{\mathrm{ch}}^{\mathrm{T}}=\left\{\psi_{R}, \psi_{L}\right\}, \hat{\sigma}^{z} \equiv \operatorname{diag}(1,-1), \psi_{R / L}$ are chiral fermionic operators of right/left moving modes, $v_{F}$ is the Fermi velocity. The Hamiltonian of the exchange interaction between the fermions and the MI is naturally written in the spin fermionic basis, $\Psi_{\mathrm{sp}}^{\mathrm{T}}=\left\{\psi_{\uparrow}, \psi_{\downarrow}\right\}$ :

$$
H_{\mathrm{int}}=\sum_{j} \sum_{k_{1,2}} J_{j} \Psi_{\mathrm{sp}}^{\dagger}\left(k_{1}\right) \hat{S}^{j} \hat{\sigma}^{j} \Psi_{\mathrm{sp}}\left(k_{2}\right), j=x, y, z ;
$$

with $\hat{S}^{j}$ and $\hat{\sigma}^{j}$ being the localized spin-1/2 operator and the Pauli matrices, respectively. The MI is located at position $x=0$. For our purposes, it suffices to assume the diagonal coupling matrix with small diagonal entries, $J_{j} \ll v_{F}$. The spin and chiral bases are related by the $k$-dependent rotation $[10,41]$

$$
\Psi_{\mathrm{sp}}(k)=\hat{B}(k) \Psi_{\mathrm{ch}}(k), \hat{B}(k) \equiv \cos (\theta(k))+i \hat{\sigma}^{y} \sin (\theta(k)) .
$$

We have introduced the angle of the spin rotation caused by the ST: $\theta(k) \equiv\left(k / k_{0}\right)^{2}$. The ST is typically weak, $k / k_{0} \sim \max \{T, e V\} / k_{0} v_{F} \ll 1$. However, using Eq.(5) [without an expansion of $\hat{B}$ in powers of $\theta(k)$ ] is technically more convenient. Eq.(4) in the chiral basis reads:

$$
\begin{aligned}
H_{\mathrm{int}} & =\sum_{k_{1,2}} \Psi_{\mathrm{ch}}^{\dagger}\left(k_{1}\right) \hat{\mathcal{G}}\left(k_{1}, k_{2}\right) \Psi_{\mathrm{ch}}\left(k_{2}\right), \\
\hat{\mathcal{G}}\left(k_{1}, k_{2}\right) & =\sum_{j} J_{j} \hat{S}^{j}\left(\hat{B}^{\dagger}\left(k_{1}\right) \hat{\sigma}^{j} \hat{B}\left(k_{2}\right)\right) .
\end{aligned}
$$

Eq.(7) shows that the ST-caused rotation by the matrix $\hat{B}$ changes (or even induces in the isotropic case) the anisotropy of the coupling. This effective anisotropy depends on the electron momentum and gives rise to the suppression of the helical conductance.

The expression for the number of the chiral electrons, which enters Eq.(1) for the backscattering current, reads:

$$
N_{\alpha}=\sum_{k} \operatorname{Tr}_{e, M I}\left\{\psi_{\alpha}^{\dagger}(k) \psi_{\alpha}(k) \hat{\rho}(t)\right\}, \alpha=R, L .
$$

Trace in Eq.(8) is calculated with respect to the helical electrons, $\operatorname{Tr}_{e}$, and the MI, $\operatorname{Tr}_{M I} ; \hat{\rho}(t)$ denotes the total (describing the electrons and the MI) density matrix in the interaction representation with respect to $H_{0}$. In the standard approach, which involves the Markovian approximation (see, e.g., the textbook [42]), $\hat{\rho}(t)$ obeys the evolution equation $\partial_{t} \hat{\rho}=-i\left[\mathcal{H}_{\text {int }}(t), \hat{\rho}(-\infty)\right]+\mathcal{L}[\hat{\rho}(t)]$, where

$$
\mathcal{L}[\hat{\rho}(t)] \equiv-\int_{-\infty}^{t} \mathrm{~d} t^{\prime}\left[\mathcal{H}_{\mathrm{int}}(t),\left[\mathcal{H}_{\mathrm{int}}\left(t^{\prime}\right), \hat{\rho}(t)\right]\right],
$$

and, in the second order in $H_{\text {int }}, \hat{\rho}$ in Eq.(9) can be factorized $\hat{\rho} \simeq \hat{\rho}_{e} \hat{\rho}_{M I}$. Here $\hat{\rho}_{e}$ is the density matrix of the free electrons coupled to the leads, $\hat{\rho}_{M I} \equiv \operatorname{Tr}_{e}\{\hat{\rho}\}$ is the reduced density matrix of the MI. The latter matrix obeys the master equation $\partial_{t} \hat{\rho}_{M I}=\operatorname{Tr}_{e}\left\{\mathcal{L}\left[\hat{\rho}_{e} \hat{\rho}_{M I}(t)\right]\right\}$ (the linear in $\mathcal{H}_{\text {int }}$ term does not contribute to $\operatorname{Tr}_{e}$ ).

The effect which we study is governed by real processes. Virtual processes lead to renormalizations, which are unimportant at $\max \{T, e V\} \gg T_{K}$. Therefore, we neglect the virtual processes and focus on the energypreserving scattering of the electrons by the MI. The master equation takes the following form

$$
\begin{aligned}
& \partial_{t} \hat{\rho}_{M I}=-\frac{\pi}{\left(2 \pi v_{F}\right)^{2}} \sum_{\alpha, \beta} \int \mathrm{d} \epsilon\left\{f_{e}^{\alpha}(\epsilon)\left[1-f_{e}^{\beta}(\epsilon)\right]\right. \\
& \left.\times\left[\left\{\hat{\mathcal{G}}_{\alpha \beta}(\epsilon) \hat{\mathcal{G}}_{\beta \alpha}(\epsilon), \hat{\rho}_{M I}\right\}_{+}-2 \hat{\mathcal{G}}_{\beta \alpha}(\epsilon) \hat{\rho}_{M I} \hat{\mathcal{G}}_{\alpha \beta}(\epsilon)\right]\right\} .
\end{aligned}
$$

Here $\alpha, \beta=R, L ; \hat{\mathcal{G}}(\epsilon) \equiv \hat{\mathcal{G}}\left(\epsilon / v_{F}, \epsilon / v_{F}\right) ; f_{e}^{R / L}$ are fermionic distribution functions:

$$
f_{e}^{R / L}(\epsilon)=\frac{1}{\exp \left[\left(\epsilon-\mu_{R / L}\right) / T\right]+1} ;
$$

$\mu_{R / L}$ are chemical potentials of the corresponding leads; we assume $\mu_{R / L}= \pm e V / 2 \Rightarrow \delta \mu \equiv \mu_{R}-$ $\mu_{L}=e V$. The stationary solution of Eq.(10), $\hat{\rho}_{M I}^{\mathrm{st}}$, determines the backscattering dc current: $\mathcal{J}_{\mathrm{dc}}=$ $2 e \operatorname{Tr}_{e, M I}\left\{\psi_{R}^{\dagger} \psi_{R} \mathcal{L}\left[\hat{\rho}_{e} \hat{\rho}_{M I}^{\mathrm{st}}\right]\right\}$. Similar to Eq.(10), we find

$$
\begin{aligned}
\mathcal{J}_{\mathrm{dc}}= & \frac{4 \pi e}{\left(2 \pi v_{F}\right)^{2}} \int \mathrm{d} \epsilon \\
& \left(f_{e}^{L}(\epsilon)\left[1-f_{e}^{R}(\epsilon)\right] \operatorname{Tr}_{M I}\left\{\hat{\mathcal{G}}_{L R}(\epsilon) \hat{\mathcal{G}}_{R L}(\epsilon) \hat{\rho}_{M I}^{\mathrm{st}}\right\}-\right. \\
& \left.-f_{e}^{R}(\epsilon)\left[1-f_{e}^{L}(\epsilon)\right] \operatorname{Tr}_{M I}\left\{\hat{\mathcal{G}}_{R L}(\epsilon) \hat{\mathcal{G}}_{L R}(\epsilon) \hat{\rho}_{M I}^{\mathrm{st}}\right\}\right) .
\end{aligned}
$$

\section{RESULTS AND THEIR DISCUSSION}

\section{Linear response}

Let us first study the linear response, $V \rightarrow 0$, and find the temperature-dependent correction to dc linear conductance, $\delta G_{L}(T) \equiv \delta G(T, V=0)$.

After a straightforward algebra, we obtain the following answers for the stationary density matrix of the MI:

$$
\begin{aligned}
\hat{\rho}_{M I}^{\mathrm{st}}= & \frac{1}{2}+\frac{\delta \mu}{T} \tilde{J}_{y} \times \\
& \times\left(\frac{\tilde{J}_{x} I_{c}(\mathcal{T})}{\tilde{J}_{x}^{2}+\tilde{J}_{y}^{2}} \hat{S}^{z}-\frac{\tilde{J}_{z} I_{s}(\mathcal{T})}{\tilde{J}_{z}^{2}+\tilde{J}_{y}^{2}} \hat{S}^{x}\right)+o(\delta \mu) ;
\end{aligned}
$$


and for $\delta G_{L}$ :

$$
\begin{aligned}
\frac{\delta G_{L}}{G_{0}}= & -\frac{1}{2} \frac{\left(J_{x}^{2}-J_{y}^{2}\right)^{2}}{J_{x}^{2}+J_{y}^{2}}+\frac{\tilde{J}_{x}^{2}-\tilde{J}_{z}^{2}}{4}\left[1-I_{c}(2 \mathcal{T})\right]+ \\
& +2 \tilde{J}_{y}^{2}\left[\frac{\tilde{J}_{x}^{2}}{\tilde{J}_{x}^{2}+\tilde{J}_{y}^{2}}\left(I_{c}^{2}(\mathcal{T})-1\right)+\frac{\tilde{J}_{z}^{2}}{\tilde{J}_{z}^{2}+\tilde{J}_{y}^{2}} I_{s}^{2}(\mathcal{T})\right] .
\end{aligned}
$$

Here $\mathcal{T} \equiv 2\left(2 T / v_{F} k_{0}\right)^{2} ; \tilde{J}_{x, y, z} \equiv J_{x, y, z} / v_{F} ; G_{0}=$ $e^{2} / h$ is the helical ballistic conductance; and we have introduced two functions.

$$
I_{c}(x)=\int_{0}^{\infty} \mathrm{d} \epsilon \frac{\cos \left(x \epsilon^{2}\right)}{\cosh ^{2}(\epsilon)}, I_{s}(x)=\int_{0}^{\infty} \mathrm{d} \epsilon \frac{\sin \left(x \epsilon^{2}\right)}{\cosh ^{2}(\epsilon)} .
$$

Since $\delta G_{L}$ depends on $T / v_{F} k_{0}$, its temperature independent part corresponds to the limit of small temperature and, simultaneously, of the vanishing ST.

If the MI is isotropic, $\tilde{J}_{x, y, z}=\tilde{J}$, Eqs. $(13,14)$ reduce to

$$
\begin{aligned}
& \hat{\rho}_{M I}^{\mathrm{st}} \simeq \frac{1}{2}+\frac{\delta \mu}{2 T}\left(I_{c}(\mathcal{T}) \hat{S}^{z}-I_{s}(\mathcal{T}) \hat{S}^{x}\right) \simeq \\
& \frac{1}{2}+\frac{\delta \mu}{2 T}\left(\left[1-\frac{14}{15}\left(\frac{\pi T}{v_{F} k_{0}}\right)^{4}\right] \hat{S}^{z}-\frac{2}{3}\left(\frac{\pi T}{v_{F} k_{0}}\right)^{2} \hat{S}^{x}\right) ; \\
& \delta G_{L}^{\text {(iso) }}=-\tilde{J}^{2}\left\{1-\left[I_{c}^{2}(\mathcal{T})+I_{s}^{2}(\mathcal{T})\right]\right\} G_{0} \simeq \\
& \simeq-\frac{4 \tilde{J}^{2} G_{0}}{45}\left(\frac{2 \pi T}{v_{F} k_{0}}\right)^{4} .
\end{aligned}
$$

Eq.(16) confirms that the isotropic (unscreened) MI is able to suppress the helical ballistic conductance in the topological insulators with broken axial spin symmetry of the electrons.

The exponent of the power-law $T$-dependence of $\delta G_{L}^{\text {(iso) }}$ is anticipated from the phase space-arguments of the above qualitative discussion and, simultaneously, the value $\delta G_{L}^{\text {(iso) }}\left(T / v_{F} k_{0}=0\right)=0$ agrees with the prediction of Ref.[32] on the vanishing effect of the isotropic MI in the absence of the ST. The temperature dependence of $\delta G_{L}^{(\mathrm{iso})} \sim\left(T / v_{F} k_{0}\right)^{4}$ dominates over that of a correction to the linear conductance, $\delta G_{\text {int }}$, governed by the combined effect of the ST and the electron interactions. $\delta G_{\text {int }}$ is parametrically smaller in a clean system and reads as either $\delta G_{\text {int }} \propto\left(T / v_{F} k_{0}\right)^{5}$ at $k_{F}=0$ or $\delta G_{\text {int }} \propto\left(v_{F} k_{0} / T\right) \exp \left(-v_{F} k_{0} / T\right)$ at $v_{F} k_{F} \gg T$ [10].

The bare XY-anisotropy of the coupling constants makes $\left.\delta G_{L}\right|_{T \rightarrow 0}$ in Eq.(14) finite (still assuming $T>T_{K}$ ) in agreement with the previous works [36-38], see comparison of the isotropic and anisotropic cases in Fig.2. Curiously, the ST can decrease the effect of the magnetic anisotropy and make $G_{L}(T=0)<G_{L}(T \neq 0)$, with $G_{L}(T)$ certainly being smaller than $G_{0}$. This happens, for instance, at $J_{y}=0$ in the range $0<J_{z}<J_{x}$, the red curve in Fig.2. If $J_{y}=0, J_{z}=J_{x} \neq 0$, the ST does not change $G_{L}$ which is finite and temperature independent, the green curve in Fig.2. In the regime $J_{y}=$
$0, J_{z}>J_{x} \neq 0$, the ST enhances the effect of the magnetic anisotropy such that $G_{L}(T=0)>G_{L}(T \neq 0)$, the blue curve in Fig.2. Thus, an unusual growth of the subballistic helical conductance with increasing the temperature might indicate the combined effect of the anisotropic MI and the ST. The $T^{4}$-dependence of the correction to the conductance can arise also in a model which describes the combined effect of the ST and the electron interactions in the presence of a potential disorder; their combined action results in the scaling $\delta G_{\text {int }+ \text { imp }} \propto\left(T / v_{F} k_{0}\right)^{4}$ at $v_{F} / L \ll T \ll v_{F} k_{F}$ ( $L$ is the systems size) [10]. However, this mechanism always leads to the inequality $G_{L}(T=0)>G_{L}(T \neq 0)$.

If one takes into account virtual processes, the coupling constants in Eqs. $(14,16)$ acquire an additional temperature dependence due to Kosterlitz-Thouless-like renormalizations which are very weak and can be safely ignored at $T \gg T_{K}[19,31]$. Moreover, in a particular case $J_{x, y}=0$, the renormalizations are absent at the level the first loop of the renormalization group. Therefore, the answer (14) is expected to approximately hold true down to ultra-low temperatures in the case of the easy z-axis anisotropy of the exchange coupling.

\section{Nonlinear response caused by isotropic MI}

Eqs. $(10,12)$ describe the combined effect of the MI and the ST on helical transport also at a finite bias. This effect does not vanish if the temperature approaches zero but the bias is finite, $T_{K} \ll e V$. In this case, the phase space, which is needed for the successive backscattering of the helical electrons with the same chirality, is provided by $\mathrm{eV}$ instead of $T$. It is reflected by a crossover of the differential conductance from the $T^{4}$-scaling to the $(e V)^{4}$ scaling. In particular, the correction to the nonlinear conductance governed by the isotropic MI reads as:

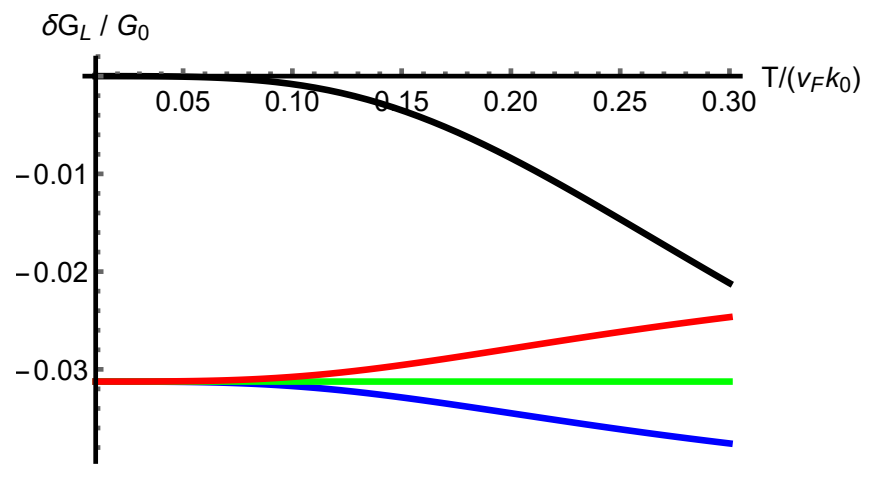

FIG. 2. Temperature dependence of the MI-generated correction to the linear ballistic helical conductance for four cases: (i) isotropic MI, $\tilde{J}_{x, y, z}=0.25$ (black curve); (ii) anisotropic MI $\tilde{J}_{x}=0.25, \tilde{J}_{y}=0, \tilde{J}_{z}=0.35$ (blue curve); (iii) easy xz-plane anisotropic MI, $\tilde{J}_{x, z}=0.25, \tilde{J}_{y}=0$ (green curve); (iv) easy x-axis anisotropic MI, $\tilde{J}_{x}=0.25, \tilde{J}_{y, z}=0$ (red curve). 


$$
\delta G^{(\text {iso })}(T \ll e V) \simeq-\frac{\tilde{J}^{2} G_{0}}{18}\left(\frac{e V}{v_{F} k_{0}}\right)^{4}=\frac{5}{8}\left(\frac{u}{\pi}\right)^{4} \delta G_{L}^{(\text {iso })} ; \quad u \equiv \frac{e V}{2 T}
$$

The crossover between regimes of the infinitesimal bias, Eq.(16), and the vanishing temperature, Eq.(17), is described by a rather complicated function:

$$
\frac{\delta G^{(\text {iso })}(T, V)}{\delta G_{L}^{(\text {iso })}(T)} \simeq 1-\frac{\tanh ^{2}(u)}{2}+\frac{15}{4}\left(\frac{u}{\pi}\right)^{2}\left[1+\frac{\tanh (u)}{u}-\frac{\tanh ^{2}(u)}{2}\right]+\frac{5}{4}\left(\frac{u}{\pi}\right)^{4}\left[1+\frac{4+2 u \operatorname{coth}(u)-u^{2}}{2(1+u \operatorname{coth}(u))^{2}}\right]
$$

see the main panel of Fig.3.

Complexity of Eq.(18) at $T \simeq e V$ is related to the MI polarization, which develops with increasing the ratio $e V / T$. For instance, the partial mean polarization of the isotropic MI along the helical edge, $S_{x}=\operatorname{Tr}\left[\hat{S}^{x} \rho_{M I}^{\mathrm{st}}\right]$, is given by

$$
\frac{S_{x}(T, e V)}{S_{x}^{(0)}(T, e V)} \simeq \frac{1+\left(\frac{u}{\pi}\right)^{2}+\left[1+3\left(\frac{u}{\pi}\right)^{2}\right] \frac{\tanh (u)}{u}}{1+u \operatorname{coth}(u)}
$$

see the inset in Fig.3. Here $S_{x}^{(0)}=-(2 u / 3)\left(\pi T / v_{F} k_{0}\right)^{2}$ is the corresponding value of the MI polarization in the linear regime, cf. Eq.(15).

\section{SUMMARY}

We have demonstrated that the spin texturing, which reflects the absence of the spin axial symmetry of electrons, noticeably changes the influence of dynamical magnetic impurities on the helical transport on edges of $2 \mathrm{D}$ topological insulators. This is particular pronounced in the emergent ability of the (unscreened) spin U(1)invariant MI to suppress the ballistic dc helical conductance. We have exemplified such a suppression by

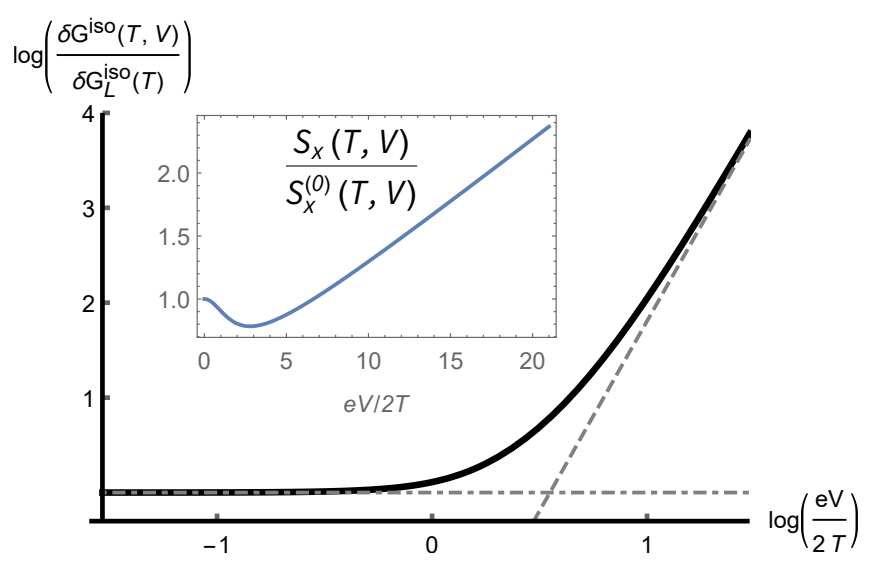

FIG. 3. Main panel: Crossover of the correction to the differential conductance, Eq.(18), between limits of the linear response, $\mathrm{eV} \ll T_{K} \ll T$ (dash-dotten line), and the finite bias, $T, T_{K} \ll e V$ (dashed line). Inset: Polarization of the MI spin along $x$-axis (i.e., along the helical edge), Eq.(19). considering the example of the isotropic MI. This effect does not require the energy transfer but, nevertheless, is temperature-dependent. The corresponding contribution to the linear helical conductance, $\delta G_{L}^{(\text {iso) }}$ in Eq.(16), is negative and its magnitude decreases as $T^{4}$ while lowering the temperature. It is related to the underlying physical mechanism of suppression of the dc conductance: the isotropic MI can backscatter one after another electrons propagating in the same direction if they have different orientation of spins due to different energies. If $T \rightarrow 0$, transport is carried by the electrons on the Fermi level, the available phase space of the electrons shrinks and the predicted effect disappears, namely, the isotropic MI does not affect the helical conductance similar to the systems with the spin axial symmetry (no ST). The predicted $T^{4}$ dependence of $\delta G_{L}^{(\text {iso) }}$ is parametrically greater than that anticipated in clean systems with the ST and the electron interactions [10].

If the applied external voltage if finite and $T \ll e V$, the available phase space is provided by the voltage and the differential conductance scales as $G^{\text {(iso) }} \propto(e V)^{4}$, Eq.(17). The crossover between two scaling regimes is described by Eq.(18). It reflects a partial polarization of the MI caused by a rather complicated competition of the applied voltage and the finite temperature.

We note also a curious feature of the combined effect of the ST and the anisotropic MI: if the bare anisotropy of the coupling between the MI and the conduction electrons is of the easy axis type (with the axis being directed along the edge of the topological insulator), the ST can effectively weaken the anisotropy and partially restore the ballisticity of the conductance. This effect could manifest itself in an unusual growth of the subballistic linear helical conductance with increasing $T$.

\section{ACKNOWLEDGMENTS}

O.M.Ye. acknowledges support from the DFG through the grant YE 157/2-3. V.I.Yu. acknowledges support from the Basic research program of HSE.

Author contributions: The authors have made an equal contribution to this paper. 
Competing interests: The authors declare no competing interests.
Data availability: No datasets were generated or analysed during the current study.
[1] M. König, S. Wiedmann, C. Brune, A. Roth, H. Buhmann, L. W. Molenkamp, X. L. Qi, and S. C. Zhang, QSH insulator state in HgTe quantum wells, Science 318, 766 (2007).

[2] M. König, M. Baenninger, A. G. F. Garcia, N. Harjee, B. L. Pruitt, C. Ames, P. Leubner, C. Brüne, H. Buhmann, L. W. Molenkamp, and D. Goldhaber-Gordon, Spatially Resolved Study of Backscattering in the QSH State, Phys. Rev. X 3, 021003 (2013).

[3] I. Knez, C. T. Rettner, S.-H. Yang, S. S. P. Parkin, L. J. $\mathrm{Du}, \mathrm{R}$. R. Du, and G. Sullivan, Observation of Edge Transport in the Disordered Regime of Topologically Insulating InAs/GaSb Quantum Wells, Phys. Rev. Lett. 112, 026602 (2014).

[4] E. M. Spanton, K. C. Nowack, L. J. Du, G. Sullivan, R. R. $\mathrm{Du}$, and K. Moler, Images of Edge Current in InAs/GaSb Quantum Wells, Phys. Rev. Lett. 113, 026804 (2014).

[5] M. Z. Hasan and C. L. Kane, Colloquium: Topological insulators, Rev. Mod. Phys. 82, 3045 (2010).

[6] X. L. Qi and S. C. Zhang, Topological insulators and superconductors, Rev. Mod. Phys. 83, 1057 (2011).

[7] S.-Q. Shen, Topological insulators: Dirac Equation in Condensed Matters (2nd ed.) (Springer, 2017).

[8] C. Wu, B. A. Bernevig, and S. C. Zhang, Helical liquid and the edge of QSH systems, Phys. Rev. Lett. 96, 106401 (2006).

[9] N. Lezmy, Y. Oreg, and M. Berkooz, Single and multiparticle scattering in helical liquid with an impurity, Phys. Rev. B 85, 235304 (2012).

[10] T. L. Schmidt, S. Rachel, F. von Oppen, and L. I. Glazman, Inelastic Electron Backscattering in a Generic Helical Edge Channel, Phys. Rev. Lett. 108, 156402 (2012).

[11] J. I. Väyrynen, M. Goldstein, and L. I. Glazman, Helical Edge Resistance Introduced by Charge Puddles, Phys. Rev. Lett. 110, 216402 (2013).

[12] N. Kainaris, I. V. Gornyi, S. T. Carr, and A. D. Mirlin, Conductivity of a generic helical liquid, Phys. Rev. B 90, 075118 (2014)

[13] J. I. Väyrynen, M. Goldstein, Y. Gefen, and L. I. Glazman, Resistance of helical edges formed in a semiconductor heterostructure, Phys. Rev. B 90, 115309 (2014).

[14] J. I. Väyrynen, D. I. Pikulin, and J. Alicea, NoiseInduced Backscattering in a QSH Edge, Phys. Rev. Lett. 121, 106601 (2018).

[15] S. Groenendijk, G. Dolcetto, and T. L. Schmidt, Fundamental limits to helical edge conductivity due to spinphonon scattering, Phys. Rev. B 97, 241406 (2018).

[16] M. McGinley and N. R. Cooper, Elastic backscattering of quantum spin Hall edge modes from Coulomb interactions with nonmagnetic impurities, Phys. Rev. B 103, 235164 (2021)

[17] Q. Meng, S. Vishveshwara, and T. L. Hughes, Spintransfer torque and electric current in helical edge states in QSH devices, Phys. Rev. B 90, 205403 (2014).

[18] P. Novelli, F. Taddei, A. K. Geim, and M. Polini, Failure of Conductance Quantization in Two-Dimensional Topological Insulators due to Nonmagnetic Impurities, Phys.
Rev. Lett. 122, 016601 (2019).

[19] J. Maciejko, Kondo lattice on the edge of a twodimensional topological insulator, Phys. Rev. B 85, 245108 (2012)

[20] V. Cheianov and L. I. Glazman, Mesoscopic fluctuations of conductance of a helical edge contaminated by magnetic impurities, Phys. Rev. Lett. 110, 206803 (2013).

[21] B. L. Altshuler, I. L. Aleiner, and V. I. Yudson, Localization at the Edge of a 2D Topological Insulator by Kondo Impurities with Random Anisotropies, Phys. Rev. Lett. 111, 086401 (2013)

[22] O. M. Yevtushenko, A. Wugalter, V. I. Yudson, and B. L. Altshuler, Transport in helical Luttinger Liquid with Kondo impurities, EPL 112, 57003 (2015).

[23] L. Kimme, B. Rosenow, and A. Brataas, Backscattering in helical edge states from a magnetic impurity and Rashba disorder, Phys. Rev. B 93, 081301 (2016).

[24] J. I. Väyrynen, F. Geissler, and L. I. Glazman, Magnetic moments in a helical edge can make weak correlations seem strong, Phys. Rev. B 93, 241301 (2016).

[25] J. I. Väyrynen and L. I. Glazman, Current Noise from a Magnetic Moment in a Helical Edge, Phys. Rev. Lett. 118, 106802 (2017).

[26] C.-H. Hsu, P. Stano, J. Klinovaja, and D. Loss, Nuclear-spin-induced localization of edge states in twodimensional topological insulators, Phys. Rev. B 96, 081405(R) (2017).

[27] V. D. Kurilovich, P. D. Kurilovich, and I. S. Burmistrov, Indirect exchange interaction between magnetic impurities near the helical edge, Phys. Rev. B 95, 115430 (2017).

[28] O. M. Yevtushenko and V. I. Yudson, Kondo Impurities Coupled to Helical Luttinger Liquid: RKKY-Kondo Physics Revisited, Phys. Rev. Lett. 120, 147201 (2018).

[29] C.-H. Hsu, P. Stano, J. Klinovaja, and D. Loss, Effects of nuclear spins on the transport properties of the edge of two-dimensional topological insulators, Phys. Rev. B 97, 125432 (2018)

[30] J.-H. Zheng and M. A. Cazalilla, Nontrivial interplay of strong disorder and interactions in QSH insulators doped with dilute magnetic impurities, Phys. Rev. B 97, 235402 (2018).

[31] J. Maciejko, C. Liu, Y. Oreg, X. L. Qi, C. Wu, and S. C. Zhang, Kondo effect in the helical edge liquid of the quantum spin Hall state, Phys. Rev. Lett. 102, 256803 (2009).

[32] Y. Tanaka, A. Furusaki, and K. A. Matveev, Conductance of a helical edge liquid coupled to a magnetic impurity, Phys. Rev. Lett. 106, 236402 (2011).

[33] O. M. Yevtushenko and A. M. Tsvelik, Chiral lattice supersolid on edges of QSH samples, Phys. Rev. B 98, 081118(R) (2018).

[34] O. M. Yevtushenko and V. I. Yudson, Protection of helical transport in QSH samples: the role of symmetries on edges, arXiv:1909.08460 [cond-mat] (2019).

[35] S. Doniach, The Kondo lattice and weak antiferromagnetism, Physica B+C 91, 231 (1977).

[36] Y. Vinkler-Aviv, D. May, and F. B. Anders, Analytical and numerical study of the out-of-equilibrium current 
through a helical edge coupled to a magnetic impurity, Phys. Rev. B 101, 165112 (2020).

[37] P. D. Kurilovich, V. D. Kurilovich, I. S. Burmistrov, and M. Goldstein, Helical edge transport in the presence of a magnetic impurity, Jetp Lett. 106, 593 (2017).

[38] V. D. Kurilovich, P. D. Kurilovich, I. S. Burmistrov, and M. Goldstein, Helical edge transport in the presence of a magnetic impurity: The role of local anisotropy, Phys. Rev. B 99, 085407 (2019).

[39] C. L. Kane and E. J. Mele, QSH effect in graphene, Phys.
Rev. Lett. 95, 226801 (2005).

[40] A. Rod, T. L. Schmidt, and S. Rachel, Spin texture of generic helical edge states, Phys. Rev. B 91, 245112 (2015).

[41] G. Dolcetto, M. Sassetti, and T. L. Schmidt, Edge physics in two-dimensional topological insulators, Riv. Nuovo Cim. 39, 113 (2016).

[42] M. Le Bellac, Quantum physics (Cambridge University Press, Cambridge, 2013). 